\title{
Grandma-forgets-to-take-her-meds disease
}

$\mathrm{I}$ t's hard to develop a good conspiracy theory these days - that is, Cone that isn't too wacko. But here's one to test drive, one involving drug companies, retail drug stores and the investors, builders and operators of long-term care housing for the elderly.

Clearly, there's a growing pile of cash waiting for those building housing, providing meals and dishing medical treatment to the elderly. This is, after all, the fastest growing demographic in Canada, with the number of people over 85 set to triple in the next 20 years. In Canada, public money has mostly left the game of housing and caring for Grandma, largely because the private market does such a fine job. And with less pesky red tape and fewer regulations, private property developers and private "care agencies" are teaming up with a host of care providers to meet the growing gamut of Grandma's needs.

Virtually every community in Canada has now sprouted new retirement villas, assisted- and independentliving condo developments and other deluxe and elegant abodes for our elders. Who would have thought that peach-coloured stucco would be in such high demand?

Finding ways to grow the market even further can begin with a peek into an older person's medicine cabinet, where you'll discover drugs to alter everything from cholesterol, blood pressure, blood sugar, mood and anxiety to libido and bone density. About $20 \%$ of Canada's population is 60 or older, and this group consumes about $60 \%$ of all prescribed treatments. Even though the average Canadian consumes a meagre 13 prescriptions per year, among the truly heavy drug users - many of whom are elderly - it's not unusual to find people routinely swallowing 30 or 40 prescribed pills per day.

Trying to manage 30 different pills a day poses some serious problems from a practical level: some are to be taken in the morning, some at midday, some be-

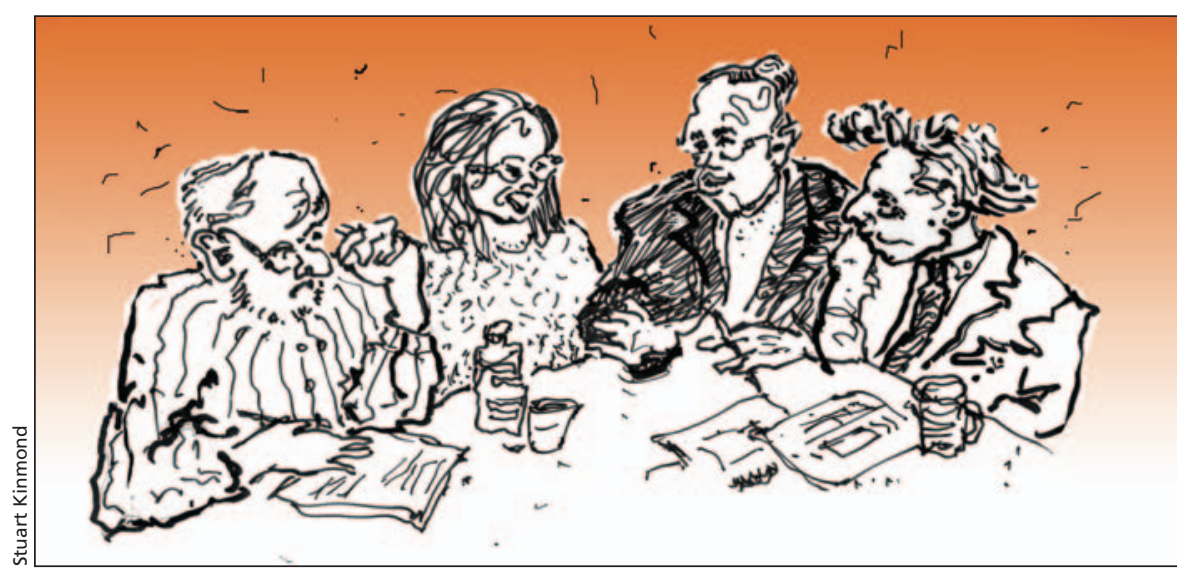

fore meals, some after meals, some before sex, etcetera, etcetera. Never mind compounding factors like mild dementia or forgetfulness. Even if your elevator goes all the way to the top, most of us would have trouble hitting the right buttons in a complex drug menu.

Some seniors use a dosette, or bubble pack, with a week's worth of medications arranged under clear plastic bubbles; this makes it easier for caregivers to determine if Grandma has been "compliant." A missed pill may signal the end of independent living for her; she may now need "medication assistance."

Whoa, I say. Before you place that call to the real estate developer, doesn't it make sense to call a trusted doctor or pharmacist first, or at least find someone who can help figure out which drugs are truly necessary (those that help Grandma feel more comfortable and able to make her own toast, for example) and those which are not? Then you can get to work on streamlining her drug-taking mess.

But that's old fashioned thinking. Today's medical care industry streamlines Grandma into "assisted living," where private-care home operators are amply set up with the most elaborate of protocols, care schedules, and mandatory social activities to treat most cases of "Grandma-forgets-to-take-hermeds" disease.

The only real side effect is sticker shock when Grandma and her kids learn the cost of all this "assistance," including medications and dispensing (which in the past were part of public long-term care). The latter fees are now a lucrative cash stream with some facilities charging \$150-\$300 a month for "medication assistance."

Yup, it's silly to think of all of this as a conspiracy, but it does amount to a pretty sweet deal for all the businesses concerned.

And Grandma? Well, she'll have lots of time now to consider how she's traded in her independence for the sake of managing 30 pills a day. But look at the bright side, at least now she's got the keys to a spanking-new stucco box and professionals are looking after her pills. Over time, one can learn to love peach.

\section{Alan Cassels BA MPA \\ Victoria, BC}

Alan Cassels, a pharmaceutical policy researcher at the University of Victoria, will tackle the issue of aging pharmaceutically in his next book. Physicians with their own Grandma story to tell can contact him at cassels@uvic.ca.

Have you got an opinion about this article? Post your views at www.cmaj.ca. Potential Salon contributors are welcome to send a query to salon@cma.ca. 